\title{
NADPH oxidase: a therapeutic target for hyperoxaluria-induced oxidative stress - an update
}

\author{
Sunil Joshi ${ }^{1} \&$ Saeed R Khan*,2 \\ ${ }^{1}$ NCF Diagnostics \& DNA Technologies, 12076 Technology Avenue Alachua, FL 32615, USA \\ ${ }^{2}$ Department of Pathology, Immunology \& Laboratory Medicine, College of Medicine, University of Florida, Gainesville, FL 32610 , \\ USA \\ *Author for correspondence: Khan@pathology.ufl.edu
}

"'to reduce the production of ROS and cellular damage associated with hyperoxaluria, a combined treatment which restores mitochondrial structure and function, prevents further mitochondrial damage and inhibits NADPH oxidase activation may prove valuable."

First draft submitted: 1 October 2019; Accepted for publication: 4 October 2019; Published online: 29 October 2019

Keywords: angiotensin II $\bullet$ NADPH oxidase • NLRP3 inflammasome • oxidative stress • peptide and nonpeptide inhibitors $\bullet$ reactive oxygen species

NADPH oxidases act as a catalyst in the formation of superoxide anion radical and production of other reactive oxygen species (ROS) such as hydrogen peroxide, which leads to oxidative stress (OS) and causes damage to macromolecules. NADPH oxidase has seven known isoforms: NOX1, NOX2, NOX3, NOX4, NOX5, DUOX1 and DUOX2. These isoforms are comprised of various core catalytic subunits: p22phox, p47phox, p67phox, p40phox, DUOX activator 1, DUOX activator, NOXA1 and NOXO1.

\section{OS \& antioxidant defense}

There is a subtle equilibrium between the ROS and the antioxidants present in the biological system. Adequate levels of ROS play an essential part as secondary messengers in numerous intracellular and intercellular signaling pathways maintaining an equilibrium. When this balance is disturbed, excess ROS leads to OS which in turn causes damage to numerous macromolecules of the cell. ROS include superoxide anion $\left(\mathrm{O}_{2}^{-\cdot}\right)$, nitric oxide radical (NO), hydroxyl radical $\left(\mathrm{OH}^{\cdot}\right)$ and hydrogen peroxide $\left(\mathrm{H}_{2} \mathrm{O}_{2}\right)$, all produced by different signaling pathways which has been discussed elsewhere in detail [1]. ROS in excess causes OS and the cells react by increasing intracellular levels of antioxidants. However, the oxidants overpower the production of these antioxidants within the biological system-resulting in the redox state in the cell becoming more oxidizing.

OS has an adverse effect on the kidneys and numerous diseases of the kidneys are affected by ROS. Excessive ROS production leads to inflammatory cell and pro-inflammatory cytokine production, further leading to initial inflammation. At this stage, an important role is played by TNF- $\alpha$ and IL- $1 \beta$ as a pro-inflammatory mediator as well as NF- $\kappa \mathrm{B}$ for transcriptional regulation of the inflammatory process. At the later stages, there is increased production of TGF- $\beta$ which coordinates the extracellular matrix synthesis.

So, in the initial stages, OS on the kidneys will lead to inflammation which in the end stages leads to the formation of abundant fibrotic tissue that will decrease organ function, leading to renal failure [2]. As reviewed by Pizzino et al., some medicines such as gentamycin, bleomycin, tacrolimus and cyclosporine increase the level of super oxides and OS by lipid peroxidation and hence are nephrotoxic. Also, the heavy metals such as $\mathrm{Cd}, \mathrm{Hg}, \mathrm{Pb}$ and As, and transition metals like $\mathrm{Fe}, \mathrm{Cu}, \mathrm{Co}$ and $\mathrm{Cr}$ are robust oxidants leading to different kinds of nephropathy as well as some types of cancers [2]. 


\section{NADPH oxidase expression}

Of interest to us in this commentary is NADPH oxidase as a therapeutic target for OS - especially in the kidneys. There are other sources of ROS such as XO, uncoupled endothelial NO synthase and mitochondrial dysfunction along with NADPH oxidase which plays a significant role in production of OS [3]. Different forms of NADPH oxidases are expressed in the kidneys as Nox1, Nox2 and Nox4 (shown to be expressed in the cortex), and Nox2, p22phox, p47phox and p67phox have been present in the thick ascending loop of Henle. Nox4 has also been shown to be present in mesangial cells and podocytes, whereas macula densa has abundance of p22phox, p47phox and p67phox subunits $[1,4]$.

Studies have shown that AT-II infusion leads to the activation of NADPH oxidase which causes increased expression of p22phox and Nox 1 in the renal cortex. When AT-II production increases abnormally, it causes vascular remodeling and endothelial dysfunction. It not only increases Nox activity but also up regulates SOD enzyme, in order to maintain a balance with increased level of ROS. When this balance is disrupted, ROS production becomes out of control, leading to pathophysiological consequences [3]. A previous in-depth review has shown that if angiotensin remains for a long time in the kidneys, it can cause activation of NADPH oxidase, increased expression of p22phox and decrease in scavenging enzyme SOD leading to renal cortical hypoxia, renal vasoconstriction and hypertension [5]. In the study, there was up regulation of Nox1 in rat cultured vascular smooth muscle cells by PDGF, Ang II and serum, whereas there is down regulation of Nox 4 in the renal cortex due to excessive AT-II. AT-II activates p67phox expression in rabbit peri-adventitial fibroblasts and the mouse aorta. These research findings discussed in the review provide an insight on the role of angiotensin on the different subunits of NADPH oxidase and the regulation of expression of NADPH oxidase in the kidneys.

\section{Antioxidants \& inhibitors of NADPH oxidase}

Antioxidants such as vitamin E ( $\alpha$-tocopherol), vitamin $\mathrm{C}$ and the combination of both have been used to eliminate OS; however, many clinical studies have shown that it is not easy to remove OS with increased levels of antioxidants as reviewed by Cifuentes-Pagano et al. [6]. This would indicate that the more favorable master plan to tackle the harmful consequences of OS is to directly access and stop the source of ROS generation, which in the majority of the cases involves different NADPH isozymes. As a matter of fact, a long search for specific inhibitors of different NADPH isozymes has been going on for more than two decades. Focusing on NADPH oxidase activity has a lot of therapeutic potential for number of diseases including chronic kidney disease, pancreatic cancer, hypertension, cystic fibrosis, Parkinson's disease, pulmonary fibrosis, acute lung injury, heart failure, ischemia/reperfusion injury and stroke to name a few [6]. There are numerous ways to inhibit NADPH oxidase isozymes due to their multiplex structure and distinct regulation of each isozyme.

OS is considered a significant contributor to oxalate and calcium oxalate crystal induced renal injury and inflammation with the involvement of NLRP3 inflammasome [7,8]. Induction of hyperoxaluria and CaOx crystal deposition in rat kidneys was associated with upregulation of genes involved in inflammasome activation, encoding for NADPH oxidase and production of crystallization modulators. Apocynin treatment caused downregulation of genes and reversed many of the changes. Hyperoxaluria in mice deficient in NLRP3 and ASC adapter protein did not produce $\mathrm{CaOx}$ crystal deposition in the kidneys [9]. Previously, we did a comprehensive review on NADPH oxidase as a therapeutic target for oxalate induced injury in kidneys and listed more than 30 inhibitors, along with their mode of action and their pharmacological effects. This review showed that chemicals involved in inhibition of ROS were more efficient than general antioxidants such as vitamin $\mathrm{E}$, which is less efficient due to various factors, including decreased bioavailability [1].

\section{Different kinds of inhibitors}

Various peptide and nonpeptide inhibitors are available which inhibit the association of NADPH oxidase complex assembly. Special attention should be aimed at p47phox or the NOXO1 subunits, other molecular subunits for therapy may be the activator such as p67phox and NOXA1 along with Rac. The goal is to develop an inhibitor with increased efficiency and specificity of binding with the protein subunits. Li et al., in their review, separated the NADPH oxidase inhibitors into two classes; peptidic inhibitors and small molecule inhibitors [10]. The peptidic inhibitors include Nox2ds-tat and NoxA1ds. Nox2ds-tat has high specificity for Nox2 isozyme with no effect on Nox1, Nox 4 or XO. It is the most extensively used isoform-specific inhibitor of Nox2 isozyme. It inhibits any interaction between Nox 2 and $\mathrm{p} 47$ phox by forming a complex with Nox 2 and preventing p 47 phox translocation to the plasma membrane. The only downside is expected poor oral bioavailability which is undergoing improvement 
with emerging technologies for better stabilization, such as peptide stapling and nanoparticle formulations. NoxA1ds inhibits the activation domain of Nox 1 activator subunit NoxA1 which is homologous to p67phox domain, thus inhibiting isozyme activation. The small molecule inhibitors include diphenylene iodonium (DPI), apocynin, VAS2870, VAS3947, GKT13690, GKT137831, ML171, ebselen and tetrahydroquinolines to name a few. DPI and apocynin have been widely utilized as traditional and broad spectrum Nox inhibitors; however, they do generate off-target effects. In many studies they have shown protective effects against numerous diseases. The triazolopyrimidine derivatives VAS2870 and VAS3947 are optimistic Nox inhibitors as they have been shown to reduce ROS in cell lines, including primary vascular endothelial and smooth muscle cells without inhibiting XO or eNOS activity. VAS2870 is known as pan-Nox inhibitor as it inhibits Nox1, Nox2, Nox4 and Nox5 [10].

\section{Current reviews \& emerging approaches}

A recent study has shown antioxidant effects of inorganic nitrite in lipopolysaccharide-induced OS. Inorganic nitrite, formerly considered harmful, was shown to be identified as an important biological NO reservoir with organ protective effects on OS and inflammation. This provides a new insight to regulate the OS and inflammatory response [11]. Another study showed the combined effect of $\mathrm{N}$-acetyl cysteine and apocynin to improve renal functions in hyperoxaluric rats by reducing the activity of NADPH oxidase and mitochondrial dysfunction. This combinatorial approach showed promising therapeutic benefits in hyperoxaluria-induced nephrolithiasis [12]. A current review by Yousefian et al. has shown that polyphenolic compounds act as modulators of NADPH oxidase [13]. The active ingredients of different plants such as berberine, thymoquinone, catechin, celastrol, resveratrol and curcumin have shown anti-inflammatory, antibacterial and antioxidants effects. Flavonoids with a hydroxyl group, a methoxy group in ortho position in ring $\mathrm{b}$ and saturated 2,3-bond in ring $\mathrm{c}$ are the most dynamic NADPH oxidase inhibitors. They showed that the most powerful natural phenolic compound recently studied as NADPH oxidase inhibitors are hesperidin and G-hesperidin [13]. Another study has shown the beneficial effect of a peptide inhibitor NF02 which potently inhibits ROS production of Nox1/Noxo1/Noxa1 complex and did not affect Nox2 and XO [14]. Naringin, a natural flavanone glycoside inhibited Nox4 expression at mRNA and protein levels in streptozotocin induced diabetic nephropathy rats and high glucose-induced podocytes demonstrating that naringin improves diabetic nephropathy [15]. Another study showed GSK2795039, a novel small molecule Nox2 inhibitor to demonstrate Nox2 inhibition in vivo in a mice model. It also showed selectivity over other NADPH oxidase isoforms, $\mathrm{XO}$ and other eNOS enzymes [16]. A recent study has shown the protective role of ER $\beta$ in renal calcium oxalate crystal formation by diminishing liver oxalate biosynthesis and renal OS mediated cell injury. ER $\beta$ prevented oxalate induced OS via transcriptional suppression of the Nox 2 by direct binding to estrogen response elements on the Nox $25^{\prime}$ promotor region [17]. To improve the efficacy and bioavailability of iodonium class of NADPH oxidase inhibitor DPI, this cohort synthesized 36 analogs to improve selectivity, solubility and functionalization of DPI. Their results showed that DPI and four analogs NSCs 740104, 751140, 734428, 737392 strongly inhibited colon cancer cell line (HT-29) growth with both 737392 and 734428 showing greater than tenfold activity against ROS production inhibiting DUOX2. They exhibited structural characteristics of iodonium-class inhibitors that affect target selectivity, and this may help in future designing of specific Nox inhibitors [18]. In a recent study of NADPH oxidase inhibitors in terms of potency, specificity and mode of action, DPI, apocynin, diapocynin, Ebselen, GKT136901 and VAS 2870 were tested on cellular and molecular assays. The results showed that DPI generated dose-dependent inhibition of all isoforms in all the assays, and all other molecules showed interesting characteristics and specificity toward different isozymes of NADPH oxidase [19]; although, the role of DPI in vivo is not very promising. As mentioned above GSK2795039 stands out as a competitive Nox2 inhibitor which has the off-target effects, specificity, mechanism of action and in vivo potency advertently addressed.

\section{Conclusion}

We need additional NADPH oxidase inhibitors to be developed which can undergo and pass clinical trials. A significant limitation in the development of NADPH oxidase therapeutics is the absence of reliable methods to measure NADPH oxidase activity in vivo. Another problem which can emerge from NADPH oxidase inhibition is the interference with neutrophil's antimicrobial defense. Gene mutations affecting the Nox2 complex lead to an immune deficiency called chronic granulomatous disease (CGD). CGD patients have defective neutrophil oxidative burst and are susceptible to recurrent infections and the development of Granuloma. Since mitochondrial protection has been shown to reduce the production of ROS and cellular damage associated with hyperoxaluria [12,20] a 
combined treatment which restores mitochondrial structure and function, prevents further mitochondrial damage and inhibits $\mathrm{NADPH}$ oxidase activation may prove valuable.

\section{Financial \& competing interests disclosure}

The authors have no relevant affiliations or financial involvement with any organization or entity with a financial interest in or financial conflict with the subject matter or materials discussed in the manuscript. This includes employment, consultancies, honoraria, stock ownership or options, expert testimony, grants or patents received or pending, or royalties.

No writing assistance was utilized in the production of this manuscript.

\section{References}

1. Joshi S, Peck AB, Khan SR. NADPH oxidase as a therapeutic target for oxalate induced injury in kidneys. Oxid. Med. Cell. Longev. 2013, 462361 (2013).

2. Pizzino G, Irrera N, Cucinotta M et al. Oxidative stress: harms and benefits for human health. Oxid. Med. Cell. Longev. 2017, 8416763 (2017).

3. Brito R, Castillo G, Gonzalez J, Valls N, Rodrigo R. Oxidative stress in hypertension: mechanisms and therapeutic opportunities. Exp. Clin. Endocrinol. Diabetes 123(6), 325-335 (2015).

4. Chabrashvili T, Tojo A, Onozato ML et al. Expression and cellular localization of classic NADPH oxidase subunits in the spontaneously hypertensive rat kidney. Hypertension 39(2), 269-274 (2002).

5. Welch WJ, Blau J, Xie H, Chabrashvili T, Wilcox CS. Angiotensin-induced defects in renal oxygenation: role of oxidative stress. Am. J. Physiol. Heart. Circ. Physiol. 288(1), H22-H28 (2005).

6. Cifuentes-Pagano E, Meijles DN, Pagano PJ. The quest for selective Nox inhibitors and therapeutics: challenges, triumphs and pitfalls. Antioxid. Redox. Signal. 20(17), 2741-2754 (2014).

7. Joshi S, Wang W, Peck AB, Khan SR. Activation of the NLRP3 inflammasome in association with calcium oxalate crystal induced reactive oxygen species in kidneys. J. Urol. 193(5), 1684-1691 (2015).

8. Joshi S, Wang W, Khan SR. Transcriptional study of hyperoxaluria and calcium oxalate nephrolithiasis in male rats: inflammatory changes are mainly associated with crystal deposition. PLoS ONE 12(11), e0185009 (2017).

9. Anders HJ, Suarez-Alvarez B, Grigorescu M et al. The macrophage phenotype and inflammasome component NLRP3 contributes to nephrocalcinosis-related chronic kidney disease independent from IL-1-mediated tissue injury. Kidney Int. 93(3), 656-669 (2018).

10. Li Y, Pagano PJ. Microvascular NADPH oxidase in health and disease. Free Radic. Biol. Med. 109, 33-47 (2017).

11. Sui Y, Tian R, Lu N. NADPH oxidase is a primary target for antioxidant effects by inorganic nitrite in lipopolysaccharide-induced oxidative stress in mice and in macrophage cells. Nitric Oxide 89, 46-53 (2019).

12. Sharma M, Kaur T, Singla SK. Role of mitochondria and NADPH oxidase derived reactive oxygen species in hyperoxaluria induced nephrolithiasis: therapeutic intervention with combinatorial therapy of N-acetyl cysteine and apocynin. Mitochondrion 27, 15-24 (2016).

13. Yousefian M, Shakour N, Hosseinzadeh $\mathrm{H}$ et al. The natural phenolic compounds as modulators of NADPH oxidases in hypertension. Phytomedicine 55, 200-213 (2019).

14. Mousslim M, Pagano A, Andreotti N et al. Peptide screen identifies a new NADPH oxidase inhibitor: impact on cell migration and invasion. Eur. J. Pharmacol. 794, 162-172 (2017).

15. Zhang J, Yang S, Li H, Chen F, Shi J. Naringin ameliorates diabetic nephropathy by inhibiting NADPH oxidase 4. Eur. J. Pharmacol. 804, 1-6 (2017).

16. Hirano K, Chen WS, Chueng AL, Dunne AA, Seredenina T et al. Discovery of GSK2795039, a novel small molecule NADPH oxidase 2 inhibitor. Antioxid. Redox. Signal. 23(5), 358-374 (2015).

17. Zhu W, Zhao Z, Chou FJ et al. The protective roles of estrogen receptor beta in renal calcium oxalate crystal formation via reducing the liver oxalate biosynthesis and renal oxidative stress-mediated cell injury. Oxid. Med. Cell. Longev. 2019, 5305014 (2019).

18. Lu J, Risbood P, Kane CT Jr, et al. Characterization of potent and selective iodonium-class inhibitors of NADPH oxidases. Biochem. Pharmacol. 143, 25-38 (2017).

19. Augsburger F, Filippova A, Rasti D et al. Pharmacological characterization of the seven human NOX isoforms and their inhibitors. Redox. Biol. 26, 101272 (2019).

20. Joshi S, Khan SR. Opportunities for future therapeutic interventions for hyperoxaluria: targeting oxidative stress. Expert. Opin. Ther. Targets 23(5), 379-391 (2019). 